\title{
Marangoni Convection in Evaporating Organic Liquid Droplets on a Nonwetting Substrate
}

\author{
Aditya Chandramohan, Susmita Dash, Justin A. Weibel, Xuemei Chen and \\ Suresh V. Garimella* \\ School of Mechanical Engineering and Birck Nanotechnology Center, \\ Purdue University, West Lafayette, Indiana 47907 USA \\ *Corresponding author: sureshg@purdue.edu, Phone: (765)-494-6209
}

\section{SUPPORTING INFORMATION}

\section{Dependence of Velocity Field on Droplet Volume}

The measured velocities inside the droplet show no discernable trend with droplet volume, within the range of volumes tested, as shown in Figure S1. At a given substrate temperature, the differences in the measured maximum velocities are either negligible for a change in volume, as in Figure S1(a) and (d), or there is a small variation without any monotonic correlation, as in Figure S1(b) and (c). 
(a)

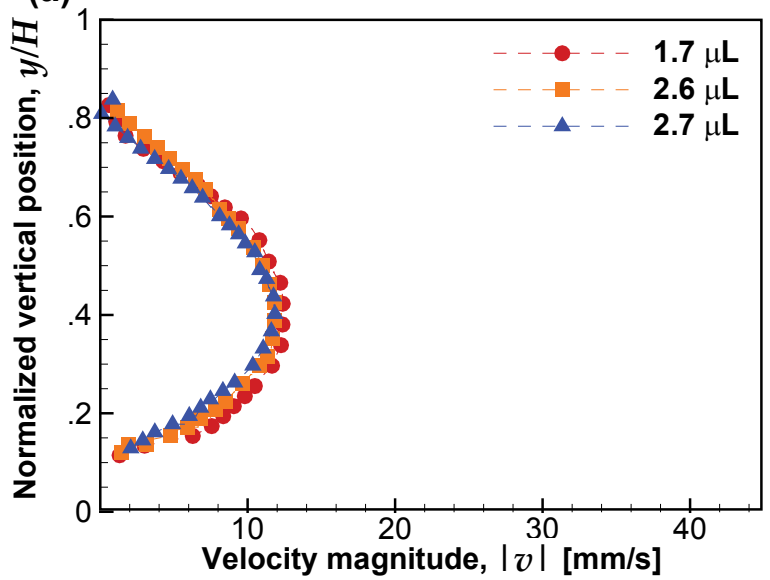

(c)

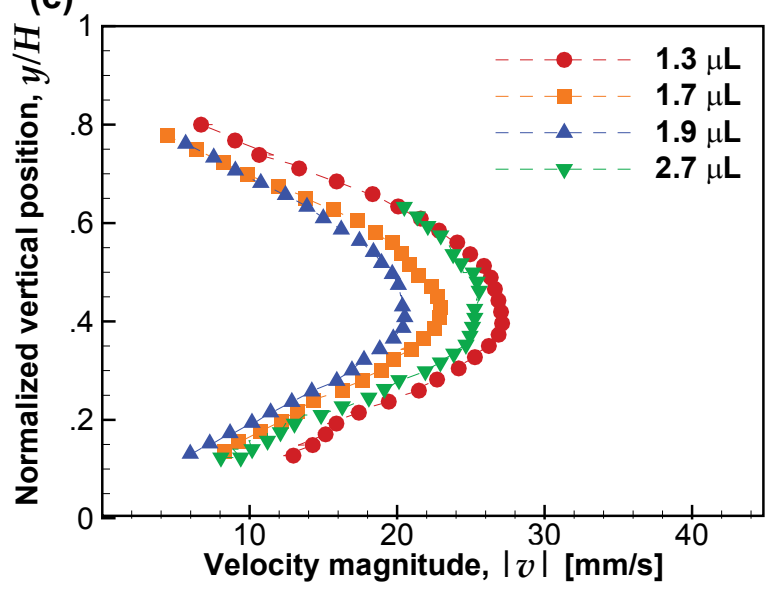

(b)

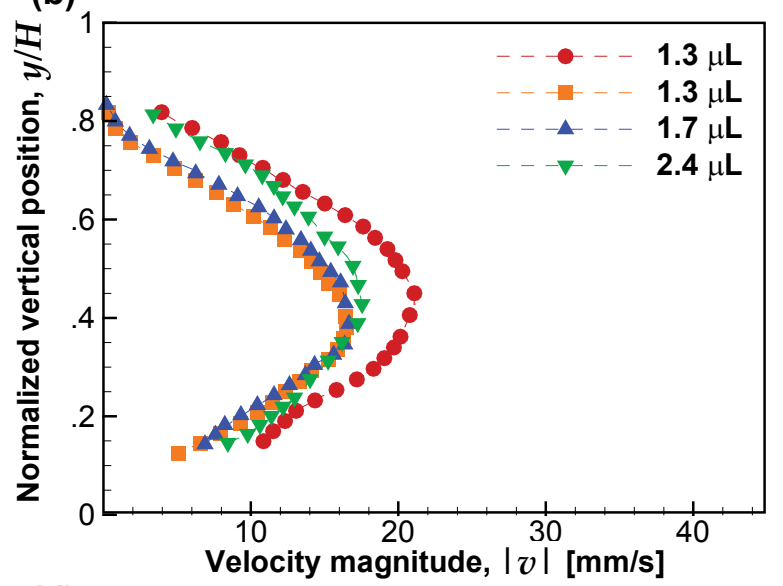

(d)

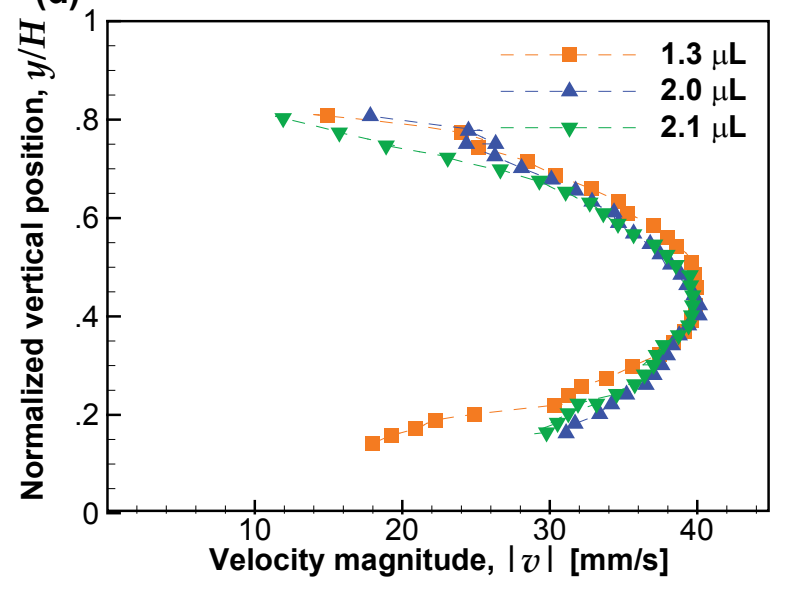

Fig. S1. The centerline vertical velocity profile is shown at multiple volumes for substrate temperatures of: (a) $5{ }^{\circ} \mathrm{C}$, (b) $10{ }^{\circ} \mathrm{C}$, (c) $15^{\circ} \mathrm{C}$, and (d) room temperature. 


\section{Marangoni Number Scaling}

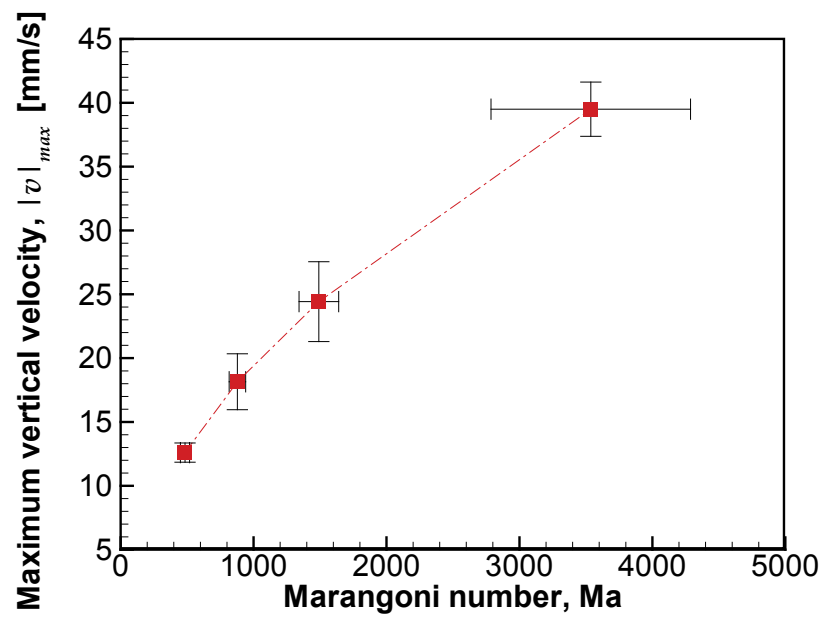

Fig. S2. Relationship between the Marangoni number and maximum velocity. The error bars represent standard deviations over all four tests at each substrate temperature $\left(5^{\circ} \mathrm{C}, 10^{\circ} \mathrm{C}, 15^{\circ} \mathrm{C}\right.$, and room temperature).

\section{Estimation of the Droplet Interface Velocity}

A comparison between the velocity data and the Marangoni velocity scale is only possible for near-interface velocities. It is necessary to estimate the interface velocity, which is masked by the curvature of the droplet, from the available data and flow field pattern. Assuming that the system is in a quasi-static state, it is possible to calculate the recirculating mass flow rate in the droplet based on the downward flow passing through a horizontal circular cross-section of the droplet. In particular, all downward velocity data are available at a horizontal cross-section passing through the vortex center. The upward flow velocities in the peripheral regions can then be estimated via mass conservation. At the same horizontal cross-section, it is known that the upward velocity is zero at the vortex center; it is assumed that the velocity increases linearly in the radial direction, as pictured in Figure S3. From this assumed upward profile, the velocity at 
the interface can be estimated based on the total recirculating mass flow rate, and compared to the scaled velocity.

(a)
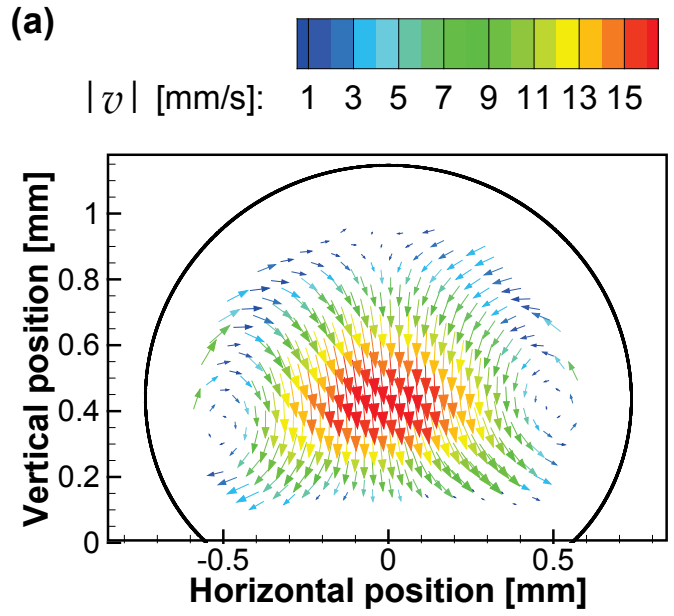

(b)

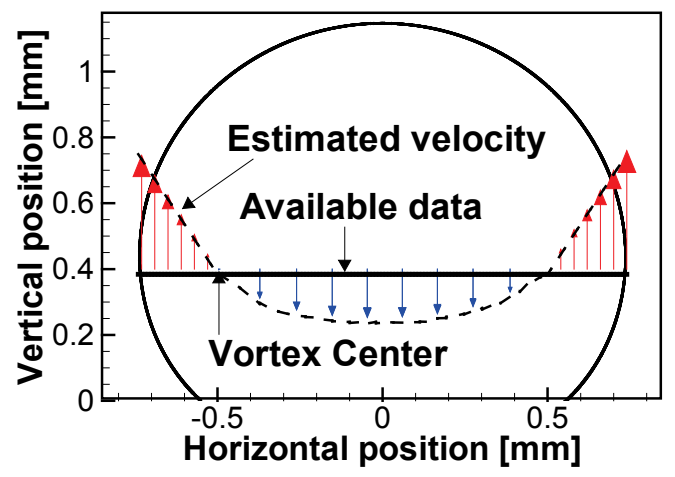

Fig. S3. (a) An example experimental velocity field (at a substrate temperature of $10{ }^{\circ} \mathrm{C}$ and droplet volume of $1.34 \mu \mathrm{L}$ ) is shown to illustrate the estimation of the near-interface upward velocity in the droplet. (b) The vertical velocity vectors are plotted at a horizontal plane of the droplet passing through the vortex center; the upward velocities in the outer region (depicted in red) are estimated based on conserving the mass flow rate from the available experimental data (depicted in blue).

\section{Reduced-Order Model Description}

Popov $^{1}$ derived an exact, closed-form solution for the evaporation of a sessile droplet via a vapor-diffusion model. The model assumed that the droplet and liquid-vapor interface are at a uniform temperature equal to the substrate temperature. For a given interface temperature, the vapor concentration at the droplet interface was assumed to be the value calculated at the saturated vapor pressure. The Laplace equation for diffusion in the concentration field was 
solved to analytically model quasi-static evaporation in the toroidal domain. The interfacial evaporation flux with radial position from the center line, $J(r)$, was defined as ${ }^{1,2}$

$$
J(r)=\frac{D\left[c_{s}\left(T_{s}\right)-\varphi c_{a}\left(T_{a}\right)\right]}{R_{c}} j(\theta)
$$

where $D$ is the diffusion coefficient, $c_{s}$ is the saturation concentration of the vapor, $T_{s}$ is the substrate temperature, $\varphi$ is the ambient relative humidity (of the droplet fluid), $c_{a}$ is the saturation concentration at the ambient temperature, $T_{a}$ is the ambient temperature, $R_{c}$ is the contact radius of the droplet, $\theta$ is the contact angle of the droplet, and $j(\theta)$ was defined as

$$
j(\theta)=\left[\frac{1}{2} \sin \theta+\sqrt{2}(\cosh \alpha+\cos \theta)^{3 / 2} \times \int_{0}^{\infty} \frac{\cosh \theta \tau}{\cosh \pi \tau} \tan [(\pi-\theta) \tau] \times P_{-(1 / 2)+i \tau}(\cosh \alpha) \tau d \tau\right]
$$

Dash and Garimella ${ }^{3}$ modified this model for evaporation of water droplets on superhydrophobic surfaces, where evaporative cooling significantly depresses the temperature of the interface due to the large thermal resistance of the droplet (due to its large height-to-diameter aspect ratio). This model incorporated one-dimensional thermal conduction along the vertical axis of the droplet to account for this effect, and predicted the interface temperature along the height of the droplet. Thus, the local evaporative flux was a function of the local interface temperature.

In the current study, a model is developed for the evaporation of organic liquid droplets on nonwetting surfaces that incorporates advective transport. The model estimates the recirculating mass flowrate in the droplet based on the Marangoni velocity, and assumes a simplified convective recirculation pattern for which coupled advection and diffusion transport is incorporated. For prediction of the temperature profile of the droplet during evaporation, the 
droplet is divided into several different regions based on the recirculating flow pattern observed in the experiments. Advective transport is considered in a central core of the droplet where the flow is downward and an outer peripheral region where the flow is upward, based on the mass conservation displayed in Figure S3(b). These regions are coupled at the top and bottom of the droplet by diffusion-dominated regions, where one-dimensional conduction is assumed in the vertical direction as in the conduction-only model of Dash and Garimella ${ }^{3}$. The model also incorporates convective heat transfer between the droplet and the ambient air. To predict a temperature drop across the height of the droplet, the substrate temperature is fixed and evaporative cooling at the interface is predicted for vapor-species diffusion to the ambient ${ }^{1,3}$. At the liquid-vapor interface, the boundary condition is a combination of evaporative cooling and convective transport to the ambient.

A few additional assumptions are necessary to simplify the transport model. It is assumed that the upward and downward flow regions do not interact (except at the top and bottom of the droplet to maintain mass continuity). The time scale of diffusion-limited evaporative mass transfer at the liquid-vapor interface ${ }^{1}, t_{D}$, is on the order of $10^{-10} \mathrm{~s}$, while the time scale of advection, $t_{A}$, is at most $0.026 \mathrm{~s}$ for methanol $\left(t_{A}=H / v\right.$, where $H$ is the droplet height and $v$ is the maximum vertical velocity for the room temperature case). Because of the drastic difference between the time scales of vapor transport and advection, the system can be assumed to be quasistatic (i.e., evaporative mass loss is neglected) in order to simplify overall mass continuity. Since it is observed that the flow velocity magnitudes are largest in the vertical direction at the height of the vortex center within the droplet core, the system mass flowrate is calculated based on the total vertical flow across this plane. Advection due to mass flow in the vertical direction is considered in the central core and outer periphery domains. In the conduction-dominated 
regions, the vertical velocity is close to zero; the horizontal velocities in this region traverse isotherms and, thus, do not contribute to the advective transport. Based on experimental evidence, this conduction-dominated region is found to be $10 \%$ of the droplet height at both the top and bottom of the droplet.

Per the simplified flow field and transport mechanisms assumed, the regions of the droplet used to construct the model are shown in Figure S4. The conduction-dominated region is discretized into a series of disk-shaped control volumes spanning the diameter of the droplet, as displayed in Figure S4(b). The energy balance in each control volume may be characterized with the following governing equation:

$$
-\left.k A_{b o t} \frac{d T}{d z}\right|_{b o t}=-\left.k A_{t o p} \frac{d T}{d z}\right|_{t o p}+J(r) h_{f g} d S+h\left(T-T_{a m b}\right) d S
$$

where $k$ is the liquid thermal conductivity, $A_{b o t}$ and $A_{\text {top }}$ are the bottom and top areas, $J(r)$ is the local evaporative flux, $h_{f g}$ is the latent heat of evaporation, $h$ is the convective heat transfer coefficient, $T$ is the temperature of the current volume, $T_{a m b}$ is the ambient temperature, and $d S$ is the infinitesimal liquid-air interface of the control volume. Note that the temperature inside each control volume is assumed to be uniform. 
(a)

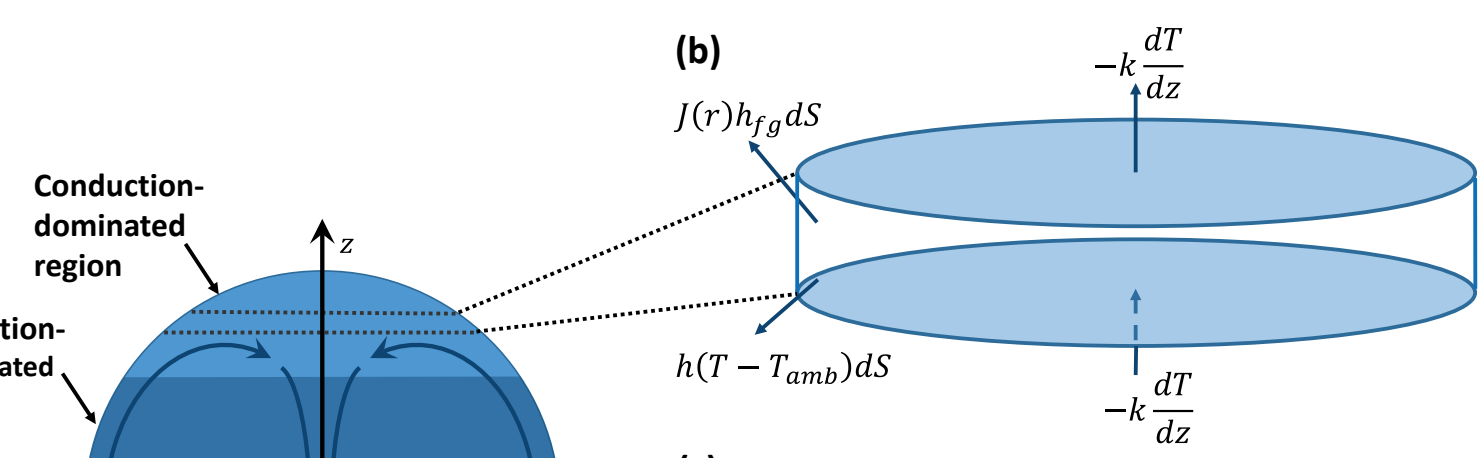

(c)

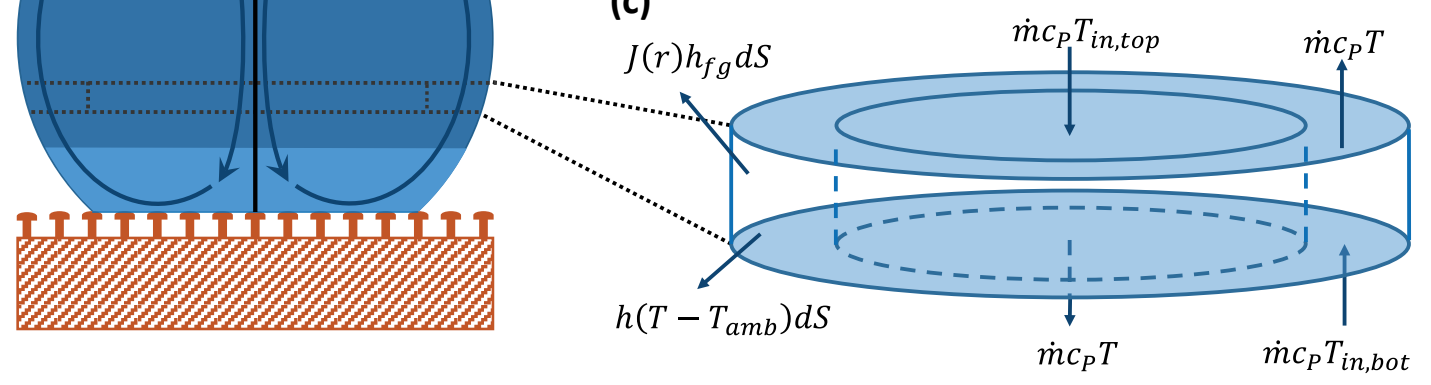

Fig. S4. (a) Schematic diagram of the evaporating droplet on a substrate with recirculating flow. The conduction-dominated and advection-dominated regions of the droplet are shown in lighter and darker shades, respectively. Representative discretized control volumes for the (b) conduction-dominated and (c) advection-dominated regions of the droplet are also shown.

In the advection-dominated region, thermal diffusion is ignored. Due to the recirculating flow, this region incorporates a cylindrical domain in the core of the droplet where the flow is downwards, and a surrounding annular domain where the flow is upwards along the interface. The discretized disk-shaped control volumes for this region are illustrated in Figure S4(c). Energy transport in the outer annular region may be defined as follows:

$$
\dot{m} c_{p} T_{b o t}=\dot{m} c_{p} T+J(r) h_{f g} d S+h\left(T-T_{a m b}\right) d S
$$


where $\dot{m}$ is the mass flowrate, $c_{p}$ is the liquid specific heat capacity, $T_{b o t}$ is the temperature from the control volume below the current one. The inner cylindrical domain uses the same equation, excluding the evaporative and convective terms on the right hand side.

The fluid properties are allowed to vary with temperature. The convective heat transfer coefficient is calculated using a Nusselt number of 2, which represents stagnant air ${ }^{4-6}$. Due to the sub-ambient temperature substrate, the convective heat transfer adds heat to the droplet. The recirculating mass flowrate is calculated using the Marangoni velocity as the interface velocity assuming the same velocity profile as described in the previous section on estimation of the droplet interface velocity. Since the temperature drop across the droplet height is not known $a$ priori, the Marangoni velocity is initialized using the temperature drop estimated via a conduction-only model ${ }^{3}$. The velocity is iterated with the temperature drop calculated using the model developed herein that includes advection.

The model is implemented in an in-house MATLAB ${ }^{7}$ code that uses a finite volume method with a first-order upwinding scheme for advective transport and first-order forward differencing for conductive transport. The energy equations are solved by iterating and updating all thermophysical properties after each iteration. Solution convergence is achieved when the control volume temperatures remain constant (within a pre-defined threshold of $10^{-7}{ }^{\circ} \mathrm{C}$ ).

\section{REFERENCES}

(1) Popov, Y. O. Evaporative Deposition Patterns: Spatial Dimensions of the Deposit. Phys. Rev. E 2005, 71 (3), 036313.

(2) Sobac, B.; Brutin, D. Thermal Effects of the Substrate on Water Droplet Evaporation. Phys. Rev. E 2012, 86 (2), 021602. 
(3) Dash, S.; Garimella, S. V. Droplet Evaporation on Heated Hydrophobic and Superhydrophobic Surfaces. Phys. Rev. E 2014, 89 (4), 042402.

(4) W. E. Ranz; W. R. Marshall. Evaporation from Drops. Chem. Eng. Prog. 1952, 48 (3), $141-146$.

(5) Levi-Hevroni, D.; Levy, A.; Borde, I. Mathematical Modeling of Drying of Liquid/Solid Slurries in Steady State One-Dimensional Flow. Dry. Technol. 1995, 13 (5-7), 1187-1201.

(6) Maitra, T.; Tiwari, M. K.; Antonini, C.; Schoch, P.; Jung, S.; Eberle, P.; Poulikakos, D. On the Nanoengineering of Superhydrophobic and Impalement Resistant Surface Textures below the Freezing Temperature. Nano Lett. 2014, 14 (1), 172-182.

(7) MATLAB Reference Manual; The Mathworks, Inc.: Natick, MA, 2007. 